\title{
Post-tracheostomy complications: respiratory failure caused by authologic foreign body-case report
}

\author{
Szymon Skoczyński ${ }^{1} \wedge$, Ewa Trejnowska ${ }^{2} \wedge$, Jarosław Paluch $^{3} \wedge$, Marcin Tomsia $^{4} \wedge$, Bartłomiej Stasiów $^{5} \wedge$, \\ Paul Armatowicz ${ }^{6} \wedge$, Ryszard Wiaderkiewicz $^{7} \wedge$, Andrzej Swinarew ${ }^{8,9}$, Kornelia Droździok ${ }^{4}$, \\ Aleksandra Oraczewska ${ }^{1 \wedge}$, Adam Barczyk $^{1 \wedge}$, Raffaele Scala $^{10} \wedge$
}

${ }^{1}$ Department of Pneumonology, Faculty of Medical Sciences in Katowice, Medical University of Silesia, Katowice, Poland; ${ }^{2}$ Department of Cardiac Anaesthesia and Intensive Care, Silesian Centre for Heart Diseases in Zabrze, Faculty of Medical Sciences in Zabrze, Medical University of Silesia, Katowice, Poland; ${ }^{3}$ Department of ENT, Faculty of Medical Sciences in Katowice, Medical University of Silesia, Katowice, Poland; ${ }^{4}$ Department of Forensic Medicine and Forensic Toxicology, Faculty of Medical Sciences in Katowice, Medical University of Silesia in Katowice, Katowice, Poland; ${ }^{5}$ The Unit of Diagnostic Imaging, Independent Public Clinical Hospital No. 7 of the Medical University of Silesia in Katowice, Professor Leszek Giec Upper Silesian Medical Centre, Katowice, Poland; ${ }^{6}$ Department of General, Endocrine and Vascular Surgery, Medical University of Warsaw, Warsaw, Poland; 'Department of Histology and Embryology, Faculty of Medical Sciences in Katowice, Medical University of Silesia, Katowice, Poland; ${ }^{8}$ Faculty of Science and Technology, University of Silesia in Katowice, Chorzów, Poland; ${ }^{9}$ Institute of Sport Science, The Jerzy Kukuczka Academy of Physical Education, Katowice, Poland; ${ }^{10}$ Department of Pulmonology and Respiratory Intensive Care Unit, S. Donato Hospital, Arezzo, Italy

Correspondence to: Szymon Skoczyński. Department of Pneumonology, Faculty of Medical Sciences in Katowice, Medical University of Silesia, 40-634 Katowice, Poland. Email: simon.mds@poczta.fm or sz.skoczynski@sum.edu.pl.

\begin{abstract}
Tracheostomy is performed frequently as a palliative treatment in patients with end-stage respiratory failure (RF). However, in patients requiring prolonged mechanical ventilation it may be difficult to recognize and can often lead to life-threatening RF. We present two cases of acute-on-chronic respiratory failure (ACRF) occurring in patients who had undergone tracheostomy [one with percutaneous dilatational tracheostomy (PDT) and the second with surgical tracheostomy (ST)]. The first case was admitted due to ACRF several months after previous successful decannulation and the second case after failure of several attempts of weaning from tracheal cannula. In both cases, noninvasive mechanical ventilation assisted flexible bronchoscopy (NIV-FB) was able to identify and solve the tracheal stenosis secondary to stiff bananashaped whitish foreign bodies. Histology sampling and genetic testing confirmed autologous foreign body formation-tracheal cartilage calcification. NIV-FB was found to be safe and effective in both diagnosis and treatment of the tracheal stenosis. Life-threatening RF connected with tracheal stenosis may be caused by rupture of tracheal cartilage ossification in patients with a history of ST and PDT. Bronchofiberoscopy performed with NIV will be a useful procedure to evaluate and treat the respiratory tract in patients with RF with suspected tracheal stenosis.
\end{abstract}

Keywords: Bronchofiberoscopy; foreign body; noninvasive mechanical ventilation; respiratory failure (RF); tracheostomy; case report

Submitted Jul 30, 2021. Accepted for publication Oct 21, 2021.

doi: $10.21037 /$ apm-21-2110

View this article at: https://dx.doi.org/10.21037/apm-21-2110

^ ORCID: Szymon Skoczyński, 0000-0003-1796-7659; Ewa Trejnowska, 0000-0003-0279-4935; Jarosław Paluch, 0000-0003-3797-5832; Marcin Tomsia, 0000-0002-4668-4685; Bartłomiej Stasiów, 0000-0001-6184-6948; Paul Armatowicz, 0000-0001-5793-0311; Ryszard Wiaderkiewicz, 00000001-7309-0121; Andrzej Swinarew, 0000-0001-6116-9510; Kornelia Droździok, 0000-0001-6715-810X; Aleksandra Oraczewska, 0000-0002-93976963; Adam Barczyk, 0000-0002-6567-9208; Raffaele Scala, 0000-0001-6448-8437. 


\section{Introduction}

Surgical tracheostomy (ST) and percutaneous dilatational tracheostomy (PDT) are frequently performed in patients requiring prolonged mechanical ventilation. Tracheostomy complications range from mild to life-threatening. The timing of complications ranges from acute to late (1).

Stenosis is usually a late complication and depending on the type of stenosis is managed either surgically or with rigid bronchoscopy (RB). Flexible bronchoscopy $(\mathrm{FB})$ is usually reserved to manage tracheostomy-related complications (i.e., airway stenosis). RB is performed in the operating theatre under general anesthesia by an expert team and is safer than not assisted FB in terms of respiratory failure (RF) risk. The risks and complications of $\mathrm{RB}$ are mainly mechanical (teeth, oropharynx, vocal cords, and bronchial injuries) or less frequently, caused by anesthesiology procedures. Conversely, FB may be complicated by RF exacerbation, which is usually caused by airway narrowing, profound sedation and only rarely mechanical injury. That is why RB is usually preferred over FB when tracheal inspection is likely to be followed by interventional endoscopic procedures. However, the choice of one over the other bronchoscopic technique should be carefully tailored to the risk-benefit balance assessment in each case; in the contest of a fragile critical patient poorly candidate to recure again to advanced intensive care unit (ICU) care. It is worth considering the feasibility and safety of a less invasive strategy based on the use of FB under appropriate non invasive respiratory assistance for the management of tracheostomy complications. However, the risks and benefits seem to favor $\mathrm{FB}$, at least in less severe cases, noninvasive mechanical ventilation assisted flexible bronchoscopy (NIV-FB) may be of help, especially for highrisk patients with acute $\mathrm{RF}$ or acute-on-chronic respiratory failure (ACRF) (2,3).

We present two cases of ACRF complicating previously performed tracheostomies (the first after and the second before decannulation). NIV-FB was successful in diagnosing post-tracheostomy tracheal stenosis caused by autologous foreign body formation. To the best of our knowledge, there has been only one study that reported the postmortal presence of ossificated tracheal cartilages (4). However, we are the first ones to describe this lifethreatening complication in living patients. We present the following two cases in accordance with the CARE reporting checklist (available at https://apm.amegroups.com/article/ view/10.21037/apm-21-2110/rc).

\section{Case presentation}

\section{ACRF}

All procedures performed in this study were in accordance with the ethical standards of the institutional and/or national research committee(s) and with the Helsinki Declaration (as revised in 2013). Written informed consent was obtained from the family of the patient for publication of this case report and accompanying images. A copy of the written consent is available for review by the editorial office of this journal.

A 70-year-old obese woman on home-NIV due to chronic RF caused by severe chronic obstructive pulmonary disease (COPD) with overlapping obstructive sleep apnea (OSA) syndrome was admitted to the pulmonary department due to severe ACRF. Two months earlier the patient was successfully de-cannulated after prolonged weaning from tracheal tubing performed with the use of the Griggs method (PDT). Due to the presence of laryngeal stridor, previous ICU stay, and poor response to combined NIV, oxygen, and pharmacotherapy, a diagnostic NIVFB was performed. RESMED ASTRAL 150 and Philips Respironics PerforMax with a bronchoscopic elbow were used. The ventilator was set in the spontaneous-timed mode (S/T) with expiratory positive airway pressure (EPAP) ranging between $8-12 \mathrm{cmH}_{2} \mathrm{O}$ and pressure support (PS) of 20-30 $\mathrm{cmH}_{2} \mathrm{O}$. Stiff foreign body fragments protruding from the tracheal mucosa and distal post-intubation stenosis, which narrowed the tracheal lumen by approximately $75 \%$, were revealed. During FB the patient was sedated with fentanyl $(100 \mu \mathrm{g})$ and midazolam $(5 \mathrm{mg})$, whereas, local anesthesia was performed with $1 \%$ xylocaine.

NIV-FB allowed for safe tracheal assessment (Figure 1) and helped to visualize lesions, which could have been missed in an intubated patient. The foreign body was removed without bleeding and sent for histopathological examination. Directly after NIV-FB, the patient was conscious with significantly decreased dyspnea and was consulted by a laryngologist and scheduled for planned laryngological treatment. However, several hours later the patient's clinical condition deteriorated rapidly due to unexpected RF exacerbation and the patient required urgent intubation, transferred to ICU and urgent laryngological management, during which no additional artificial bodies were witnessed. Unfortunately, after 8 days of treatment in the ICU the patient died due to cardiac arrest in mechanism of asystole. Histological examination revealed bone tissue. Taking into account the patient's age and acute 

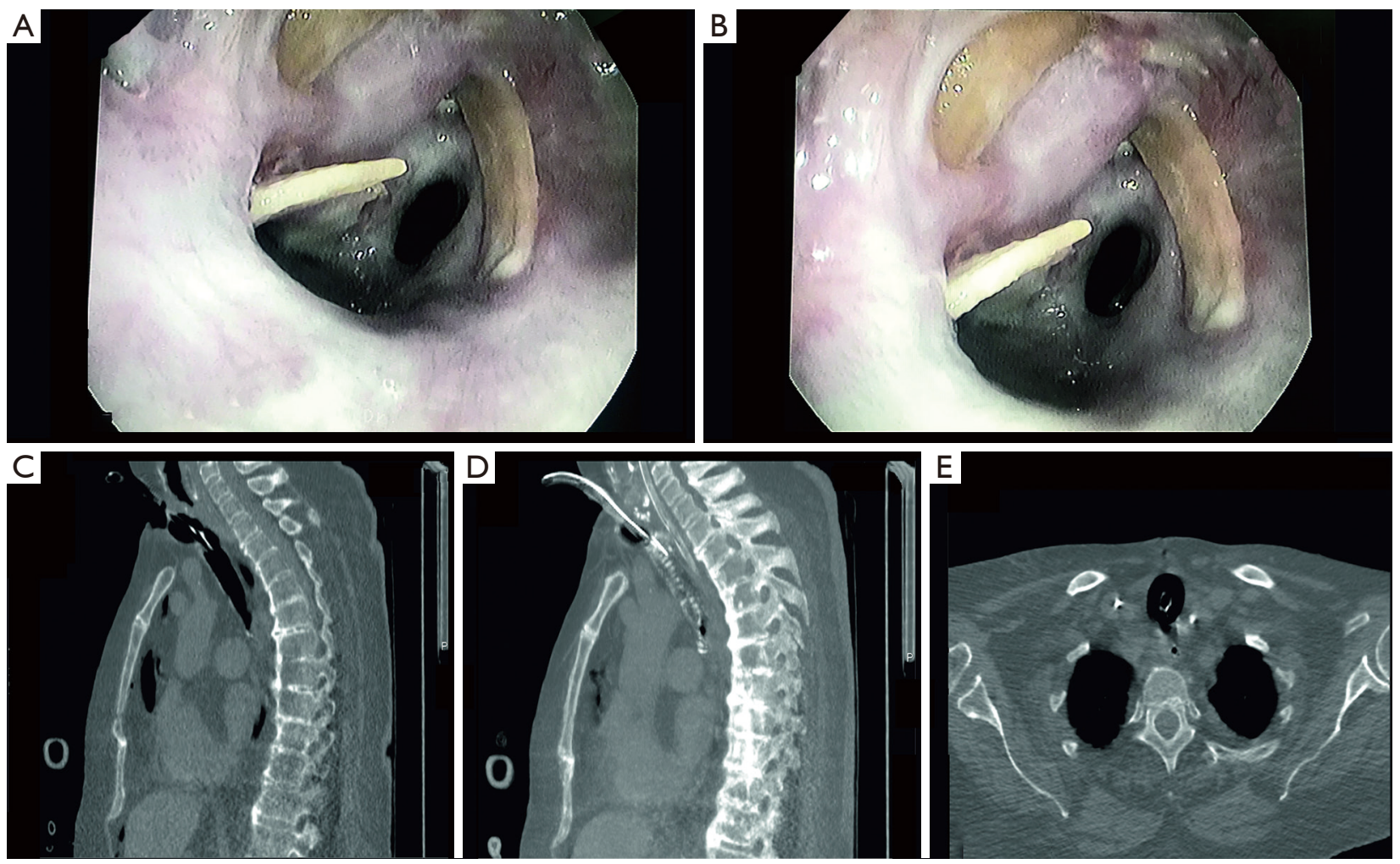

Figure 1 Bronchoscopic and CT assessment of female patient airways. (A) Bronchoscopic view of the patient's trachea (after Griggs technique), distally post-intubation tracheal stenosis can be seen. (B) Bronchoscopic view of the patient's trachea (after Griggs technique), proximally there are three pieces of foreign body in the trachea. (C) Chest-CT sagittal view: the tracheal lumen is being presented. Tracheal lumen with intubation tube placed through the trachomatous orifice. (D) Chest-CT sagittal view: the tracheal wall is being presented. In the proximal part, the tracheal cartilages are missing. Intubation tube placed into the tracheal lumen by the trachomatous orifice. (E) ChestCT transverse view. At the level of the intubation tube, there are no tracheal cartilages. (A,B) Photos performed during NIV-FB in The Department of Pneumonology in Leszek Giec Upper-Silesian Medical Centre of the Silesian Medical University in Katowice, Poland. (C-E) Chest CT performed in The Department of Diagnostic Imaging Leszek Giec Upper-Silesian Medical Centre of the Silesian Medical University in Katowice, Poland. CT, computed tomography; NIV-FB, noninvasive mechanical ventilation assisted flexible bronchoscopy.

deterioration, it was speculated that the ACRF responsible for current hospital admission was probably caused by accidental food aspiration which has taken place before described hospitalization. We could reasonably speculate post-procedural deterioration of respiratory condition without physical signs of abdomen distension should be attributed to other unpredictable causes. Even if gastric distension is a relatively frequent complication of NIV, it is likely to occur after prolonged NIV sessions in highrisk patients (i.e., mandatory supine decubitus, pre-existent oesophageal-gastro-intestinal diseases, drug use that reduces gastroenteric motility).

\section{Weaning failure: diagnosis and management}

A 68-year-old heavy smoker (40-pack-year) tracheotomized with ST method due to acute life-threatening RF of unknown etiology was transferred from ICU to the pulmonary department after 32 days of mechanical ventilation for an additional weaning attempt. Previous weaning attempts were unsuccessful due to respiratory distress. FB introduced through the tracheostomy tube showed only major carina wounding caused by airway management. The tracheostomy was removed and FB converted into trans-oral NIV-FB with the following settings: average volume assured pressure support (AVAPS), 

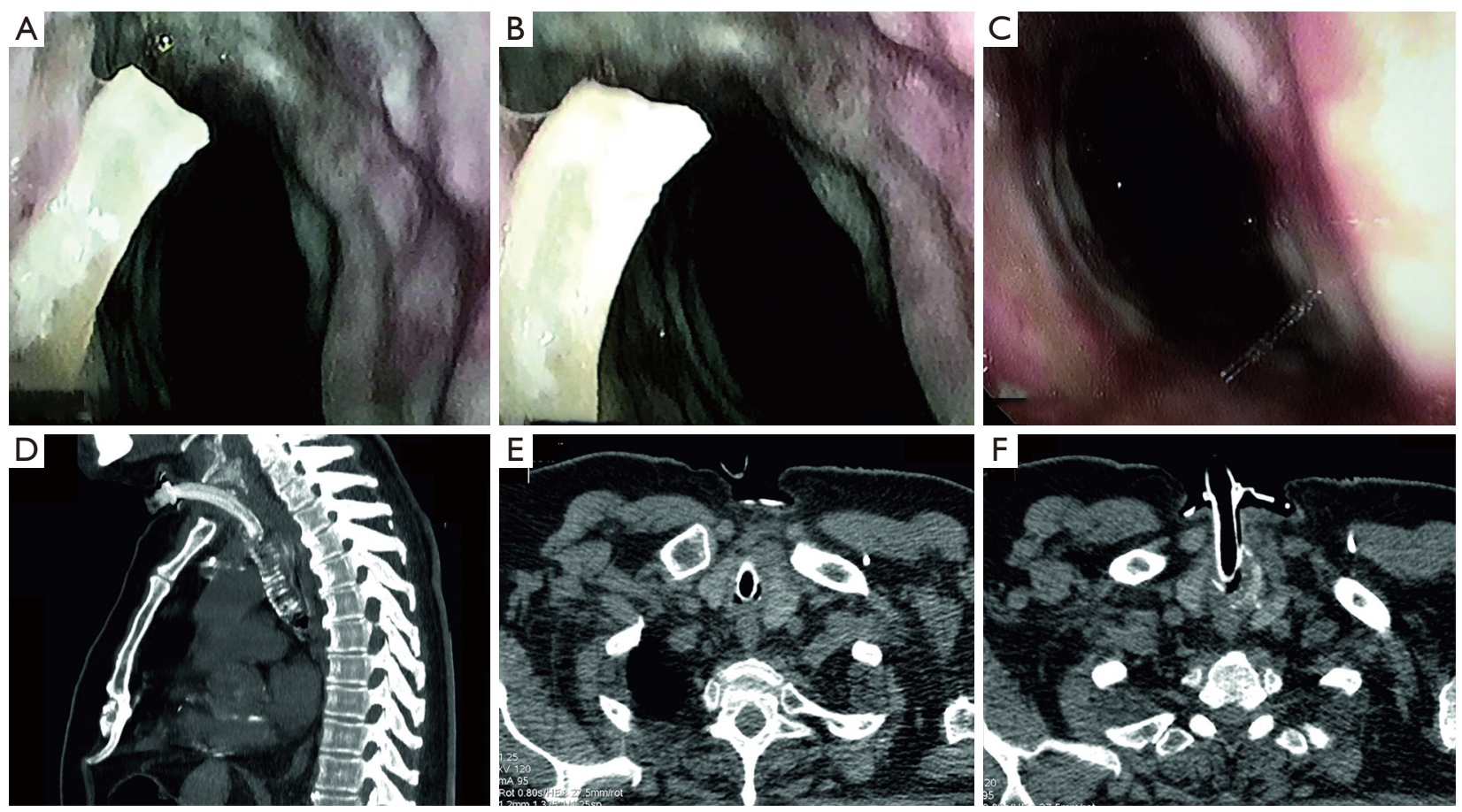

Figure 2 Bronchoscopic and CT assessment of male patient airways. (A) Bronchoscopic view of the patient's trachea (surgical tracheotomy). Subglottic granulomatous tissue and foreign body protruding from the tracheal wall and the post trachomatous orifice can be seen. (B) Subglottic granulomatous tissue and foreign body protruding from the tracheal wall. (C) The patient's trachea after foreign body removal. Post trachomatous orifice wound already closed by the occlusive dressing. (D) Chest-CT sagittal view. MIP of the trachea and placed tracheostomy tube. In the proximal part, the tracheal cartilages are missing. (E) Chest-CT transverse view at the level of the tracheostomy tube. There is tracheal lumen narrowing, but no tracheal cartilages can be seen. (F) Chest-CT transverse view at the level of the tracheostomy tube entry into the trachea. There is tracheal lumen narrowing, but only pieces of tracheal cartilages can be seen. (A-C) Photos performed during NIV-FB in The Department of Pneumonology in Leszek Giec Upper-Silesian Medical Centre of the Silesian Medical University in Katowice, Poland. (D-F) Chest CT performed in The Department of Diagnostic Imaging Leszek Giec Upper-Silesian Medical Centre of the Silesian Medical University in Katowice, Poland of the Silesian Medical University in Katowice. CT, computed tomography; MIP, maximum intensity projection; NIV-FB, noninvasive mechanical ventilation assisted flexible bronchoscopy.

tidal volume (TV) $500 \mathrm{~mL}$, PS $25-32 \mathrm{cmH}_{2} \mathrm{O}$, EPAP $8 \mathrm{cmH}_{2} \mathrm{O}$, backup rate $18 / \mathrm{min}$, inspiration time (Tins) $0.8 \mathrm{~s}$, and oxygen supplementation of 3-8 L/min. Thanks to NIVFB it was possible to visualize and subsequently remove bone fragments from the subglottic area (Figure 2). This allowed to partially restore the tracheal lumen and wean the patient from the tracheostomy.

\section{Computed tomography (CT)}

Based on the observed shape of removed bone fragments, it has been suspected that they may be composed of ossificated tracheal cartilage. However, food aspiration in severely sick patients with respiratory muscle weakness could not be excluded. Therefore, CT-scans were re-analyzed and revealed that the tracheal cartilage scaffold, which was not reported previously, was incomplete in both patients (Figures 1,2).

\section{Genetic analysis}

Genetic analysis was carried out to determine the origin of the obtained tissue and their individual identification. DNA from the reference samples and bone fragments were extracted using a Sherlock AX kit from A\&A Biotechnology (Gdynia, Poland). DNA concentrations were determined by real-time PCR using the Quantifiler ${ }^{\mathrm{TM}}$ Trio DNA Quantification Kit (AB) and the 7500 Real-Time PCR Systems AB. Template DNA was subjected to PCR reactions with kits: Power Plex ${ }^{\circledR}$ ESX 17 System, Power Plex ${ }^{\circledR} 16$ HS System PROMEGA (Madison, WI, USA) 
and AmpFISTR ${ }^{\mathrm{TM}}$ YfilerPCR Amplification Kit Applied Biosystems by Thermo Fisher Scientific (Foster City, CA, USA). Automatic analysis was carried out in $3130 \mathrm{ABI}$ Prism Applied Biosystems by Thermo Fisher Scientific. Genetic analysis of removed foreign bodies confirmed compliance with patient genotypes.

\section{Discussion}

\section{Major findings}

According to our best knowledge, we are the first to describe patients with life-threatening RF caused by tracheal stenosis secondary to autologous foreign body, which were effectively diagnosed and treated by NIV-FB. We were only able to find one report describing tracheal cartilage ossification in post-tracheostomized patients. In contrast to our observation on living patients, van Heurn et al. (4) described their cases on cadavers. The authors concluded that tracheal ossification started no earlier than 3 weeks after PDT cannulation. In our study, the post-tracheostomy complication was described in both techniques (PDT and ST). It was reported that denuded tracheal cartilage may appear in patients after repeated intubations. This type of intubation related trauma, together with mucosa swelling and mucous tissue granulation could be possibly responsible for, similar to described in our cases, tracheal stenosis (5). According to current guidelines, tracheostomy should not be performed before the fourth day of mechanical ventilation (6). It has to be underlined that in our PDT case as well as in the case described by Thompson et al. (5), RF required multidisciplinary team management which was unfortunately unsuccessful.

Based on our observations, both PDT and ST may cause autologous foreign body development and subsequent tracheal stenosis. It is estimated that tracheal stenosis is responsible for $1 \%$ of readmissions and has a mortality rate of $7.9 \%(7,8)$. Taking into account that only $6 \%$ of weaned patients manifest subjective respiratory symptoms, whereas tracheal stenosis occurs in $31 \%$ of patients (7), tracheal stenosis screening should be recommended.

To date, it is only recommended to perform FB before and during percutaneous tracheotomy. It is not recommended to perform $\mathrm{FB}$ at the time of decannulation. However, these guidelines suggest that a multidisciplinary decannulation protocol should be available in ICU's (6). This approach may probably only decrease the risk of early post-tracheostomy complications. In our opinion, to increase decannulation safety multidisciplinary management should take place both at the time of tracheostomy performance, as well as at the time of decannulation $(5,6)$. Tracheostomy seems to be the only rescue measure for a patient with severe tracheal stenosis.

Once the diagnosis is confirmed, the patient should undergo a tracheostomy to prevent further hypoxia (9). Similarly, to Shen et al. (9), we have found that tracheal stenosis qualified as a late tracheostomy complication is a life-threatening condition, in which even ICU treatment may not be sufficient enough to save the patient's life. Looking back on our case with ACRF and on the case described by Shen et al. (9), severe tracheal narrowing due to high risk of death even in clinically stable should undergo urgent laryngological management because life-threatening RF may appear rapidly (9).

In contrast to Lim et al. (10), our cases were assessed after tracheostomy was performed. Therefore, it is impossible to tell whether our findings were caused only by tracheostomy and secondary ossification or if the complication (we did not perform a complication!) arouse in a patient with previous tracheobronchopathia osteochondroplastica (10), taking into account that the age of typical tracheobronchopathia osteochondroplastica subjects is similar to our patients (11).

Similarly, to Norwood et al. (7), we found that chestCT may be useful (i.e., identification of missing tracheal cartilage) for tracheal stenosis screening in the highrisk population. However, due to the radiation burden, we cannot routinely recommend CT in all tracheostomy patients that have been weaned. Spirometry with pressurevolume curves represents a specific, but not sensitive screening tool dedicated only to asymptomatic patients (12).

Although confirmed on a younger and different population group, ultrasound may be considered another noninvasive promising tool which could replace $\mathrm{CT}$ in tracheal imaging (13). Therefore, further studies are required to reveal whether ultrasound may be considered a screening tool for tracheal stenosis in high-risk populations. However, this is currently being assessed only in the pediatric population (14).

An additional and very important novelty of our study is the role of NIV-FB in the diagnosis and management of this type of post-tracheostomy complication. There is a considerable risk of unassisted FB for patients with RF (2). Hence, RB is still most frequently used for the management of tracheal stenosis. However, there is growing evidence that NIV could be considered both a diagnostic and therapeutic tool for safe FB performance in patients 
with other causes of RF (15). In conclusion, although both patients were in severe clinical condition, NIV-FB was performed and found to be safe. To facilitate NIV-FB in both patients we have used our standard strategy, which is to begin with EPAP settled at the level of 6-8 $\mathrm{cmH}_{2} \mathrm{O}$ and subsequently adjust EPAP pressure based on patients' clinical status and observed vital and respiratory parameters but keeping the EPAP $\leq 14 \mathrm{cmH}_{2} \mathrm{O}$ which together with inspiratory time $<1 \mathrm{~s}$ decreases the risk of gastric distension but facilitates sufficient alveolar recrutation (16).

We are aware that the major limitation of our observation is the fact that it is based on only two cases. However, during the COVID-19 pandemic, when the likelihood for ICU transfer is at the level of $26.1 \%$ with mechanical ventilation need of $12.3 \%$ (17) and the tracheostomy risk as high as $13 \%(18)$, there is a considerable risk that the number of tracheotomized patients will substantially increase. Due to large occurrence of COVID-19 related stenosis preventive, diagnostic and therapeutic strategies are developed to facilitate treatment of this increasing group of patients (19). For this reason, the message of our report is likely to be of high clinical relevance.

In our cases, we did not expect to find tracheal narrowing partially caused by a foreign body. During NIVFB the foreign body was separated into small parts which looked safe for removal, but created a significant risk of transposition and closing of adjacent post intubation tracheal stenosis (Figure 1). Therefore, considering stabile ventilation during NIV-FB we were successful to clear the trachea to the maximum possible extent. Our case series would like to show in clinical ground potential rescue solutions in unpredicted circumstances occurring in fragile critical patients poorly candidates to advanced invasive treatment especially in centers with limited access to RB; this does not mean NIV-FB represents the golden standard strategy to manage life-threatening foreign body removal.

Tracheal stenosis with life-threatening RF may be caused by ruptured tracheal cartilage ossification in patients with a history of ST and PDT. NIV-FB may be useful for airway assessment and treatment in patients with RF and suspected tracheal stenosis, however RB should be available to perform safely interventional procedures under assisted ventilation and full airway control.

\section{Acknowledgments}

We would like to acknowledge the patients' relatives for their permission to publish the CT scans of their parents, who have sadly passed away between their treatment and the time of publication.

Funding: The work was supported by the Silesian Medical University (PCN-1-008/N/0/K).

\section{Footnote}

Reporting Checklist: The authors have completed the CARE reporting checklist. Available at https://apm.amegroups. com/article/view/10.21037/apm-21-2110/rc

Peer Review File: Available at https://apm.amegroups.com/ article/view/10.21037/apm-21-2110/prf

Conflicts of Interest: All authors have completed the ICMJE uniform disclosure form (available at https://apm. amegroups.com/article/view/10.21037/apm-21-2110/coif). The authors have no conflicts of interest to declare.

Ethical Statement: The authors are accountable for all aspects of the work in ensuring that questions related to the accuracy or integrity of any part of the work are appropriately investigated and resolved. All procedures performed in this study were in accordance with the ethical standards of the institutional and/or national research committee(s) and with the Helsinki Declaration (as revised in 2013). Written informed consent was obtained from the family of the patient for publication of this case report and accompanying images. A copy of the written consent is available for review by the editorial office of this journal.

Open Access Statement: This is an Open Access article distributed in accordance with the Creative Commons Attribution-NonCommercial-NoDerivs 4.0 International License (CC BY-NC-ND 4.0), which permits the noncommercial replication and distribution of the article with the strict proviso that no changes or edits are made and the original work is properly cited (including links to both the formal publication through the relevant DOI and the license). See: https://creativecommons.org/licenses/by-nc-nd/4.0/.

\section{References}

1. Spataro E, Durakovic N, Kallogjeri D, et al. Complications and 30-day hospital readmission rates of patients undergoing tracheostomy: A prospective analysis. Laryngoscope 2017;127:2746-53.

2. Esquinas A, Zuil M, Scala R, et al. Bronchoscopy during 
non-invasive mechanical ventilation: a review of techniques and procedures. Arch Bronconeumol 2013;49:105-12.

3. Chawla R, Dixit SB, Zirpe KG, et al. ISCCM Guidelines for the Use of Non-invasive Ventilation in Acute Respiratory Failure in Adult ICUs. Indian J Crit Care Med 2020;24:S61-81.

4. van Heurn LW, Theunissen PH, Ramsay G, et al. Pathologic changes of the trachea after percutaneous dilatational tracheotomy. Chest 1996;109:1466-9.

5. Thompson G, Harrison M, Ahmad H. Management of a Firmly Lodged Endotracheal Tube: A Case of High Quality Communication. J Coll Physicians Surg Pak 2021;30:582-4.

6. Trouillet JL, Collange O, Belafia F, et al. Tracheotomy in the intensive care unit: guidelines from a French expert panel. Ann Intensive Care 2018;8:37.

7. Norwood S, Vallina VL, Short K, et al. Incidence of tracheal stenosis and other late complications after percutaneous tracheostomy. Ann Surg 2000;232:233-41.

8. Johnson RF, Saadeh C. Nationwide estimations of tracheal stenosis due to tracheostomies. Laryngoscope 2019;129:1623-6.

9. Shen Y, Li K, Chen P, et al. Asphyxia caused by delayed subglottic stenosis after neck trauma. Forensic Sci Med Pathol 2021;17:481-5.

10. Lim SY, Abu Samah MF, Pereirasamy L, et al. Tracheobronchopathia osteochondroplastica-stalactite of airways. Respirol Case Rep 2021;9:e00790.

11. Ulasli SS, Kupeli E. Tracheobronchopathia osteochondroplastica: a review of the literature. Clin Respir J 2015;9:386-91.

Cite this article as: Skoczyński S, Trejnowska E, Paluch J, Tomsia M, Stasiów B, Armatowicz P, Wiaderkiewicz R, Swinarew A, Droździok K, Oraczewska A, Barczyk A, Scala R. Post-tracheostomy complications: respiratory failure caused by authologic foreign body-case report. Ann Palliat Med 2022;11(8):2761-2767. doi: 10.21037/apm-21-2110
12. Nouraei SM, Patel A, Virk JS, et al. Use of pressurevolume loops for physiological assessment of adult laryngotracheal stenosis. Laryngoscope 2013;123:2735-41.

13. Diwakar A, Adam RJ, Michalski AS, et al. Sonographic evidence of abnormal tracheal cartilage ring structure in cystic fibrosis. Laryngoscope 2015;125:2398-404.

14. Bell JR, Cohen AP, Graff JT, et al. Pilot Study to Assess the Use of Ultrasound in Evaluating the Abnormal Pediatric Airway. Otolaryngol Head Neck Surg 2020;162:950-3.

15. Skoczyński S, Minarowski Ł, Tobiczyk E, et al. Noninvasive Ventilation-Facilitated Bronchofiberoscopy in Patients with Respiratory Failure. Adv Exp Med Biol 2019;1160:53-64.

16. Skoczyński S, Ogonowski M, Tobiczyk E, et al. Risk factors of complications during noninvasive mechanical ventilation -assisted flexible bronchoscopy. Adv Med Sci 2021;66:246-53.

17. Wang D, Hu B, Hu C, et al. Clinical Characteristics of 138 Hospitalized Patients With 2019 Novel CoronavirusInfected Pneumonia in Wuhan, China. JAMA 2020;323:1061-9.

18. Abe T, Madotto F, Pham T, et al. Epidemiology and patterns of tracheostomy practice in patients with acute respiratory distress syndrome in ICUs across 50 countries. Crit Care 2018;22:195.

19. Piazza C, Filauro M, Dikkers FG, et al. Long-term intubation and high rate of tracheostomy in COVID-19 patients might determine an unprecedented increase of airway stenoses: a call to action from the European Laryngological Society. Eur Arch Otorhinolaryngol 2021;278:1-7. 